\title{
Calidad del servicio, satisfacción y fidelización
}

\author{
Quality of service, satisfaction and loyalty \\ Qualidade de serviço, satisfação e fidelidade
}

Recibido: mayo 2019

Arbitrado: junio 2019

Publicado: septiembre 2019
《 Walter Aramayo Mendoza

aramayomendozawalter@gmail.com

Universidad Andina Simón Bolívar, Bolivia

\section{RESUMEN}

Saber fidelizar clientes permite diferenciarse en mercados altamente competitivos. Por lo que el propósito de este estudio es diseñar una estrategia de fidelización para una empresa de venta de vehículos en la ciudad de Sucre, Bolivia. La investigación tuvo alcance relacional, aplicó el modelo Servperf para medir la satisfacción, la prueba chi-cuadrado para determinar la relación entre satisfacción y fidelización. Por su parte, la estrategia fue diseñada siguiendo el proceso ARC (administración de relaciones con los clientes). Como resultado se probó que existe relación directa entre satisfacción de servicios (en 5 dimensiones) y fidelización, asimismo se propuso una estrategia de fidelización enfocada principalmente en intensificar relaciones con los clientes. Se concluye que la venta de vehículos nuevos puede potenciarse elevando el componente de servicios, diferenciándose así de la competencia y fidelizando a los clientes.

Palabras clave: Calidad de servicio; fidelización; satisfacción del cliente; creación de valor; marketing relacional, bases de datos
Knowing how to retain customers allows you to differentiate yourself in highly competitive markets. Therefore, the purpose of this study is to design a loyalty strategy for a vehicle sales company in the city of Sucre, Bolivia. The research had a relational scope, it applied the Servperf model to measure satisfaction, the chisquare test to determine the relationship between satisfaction and loyalty. For its part, the strategy was designed following the ARC process (customer relationship management). As a result, it was proven that there is a direct relationship between service satisfaction (in 5 dimensions) and loyalty, and a loyalty strategy focused mainly on intensifying relationships with customers was proposed. It is concluded that the sale of new vehicles can be boosted by increasing the service component, thus differentiating itself from the competition and building customer loyalty.

Key words: Quality of service; loyalty; customer satisfaction; value creation; relationship marketing, databases
RESUMO

Saber como reter clientes permite que você se diferencie em mercados altamente competitivos. Portanto, o objetivo deste estudo é traçar uma estratégia de fidelização para uma empresa de vendas de veículos na cidade de Sucre, Bolívia. A pesquisa teve um escopo relacional, aplicou-se o modelo Servperf para medir a satisfação, o teste do quiquadrado para determinar a relação entre satisfação e lealdade. Por sua vez, a estratégia foi desenhada seguindo o processo ARC (gestão de relacionamento com o cliente). Como resultado, foi comprovado que existe uma relação direta entre a satisfação com o serviço (em 5 dimensões) e a fidelização, sendo também proposta uma estratégia de fidelização centrada principalmente na intensificação do relacionamento com os clientes. Conclui-se que a venda de viaturas novas pode ser potenciada aumentando a componente de serviço, diferenciando-se da concorrência e fidelizando o cliente.

Palavras chave: Qualidade de serviço; lealdade; satisfação do cliente; Criação de valor; marketing de relacionamento, bancos de dados 


\section{INTRODUCCIÓN}

$\mathrm{E}$ n el ámbito empresarial, el saber fidelizar a los clientes permite diferenciarse de la competencia. Este proceso empieza por escuchar al cliente, ayuda a reorientar el negocio con eficiencia y generando valor real, para traducirse en rentabilidad y posicionamiento, a la vez de ser una herramienta práctica para atraer a nuevos clientes, apoyándose en la publicidad boca en boca, (EAE, 2017).

El producto que comercializa una empresa siempre va acompañado de servicios, que constituyen un aporte en la construcción de la propuesta de valor, es importante analizar el servicio en las diferentes áreas de una empresa, a través de la percepción del cliente, (Kotler, 2006). En este sentido, la magnitud de participación del componente servicio en la venta de productos tangibles es muy variable, nótese que, en el caso de la comercialización de vehículos, los servicios agregados generan alto nivel de diferenciación y afectan considerablemente el grado de satisfacción del cliente. Es más barato retener a clientes actuales que persuadir a nuevos clientes, (Serrano, 2013)

La comercialización de vehículos nuevo ha crecido considerablemente en estos últimos años a nivel mundial, situación que se reflejó en Bolivia gracias al ingreso de multinacionales. En los últimos años el mercado se ha vuelto más competitivo y algunas empresas empezaron a apostar por los servicios complementarios para lograr fidelizar a sus clientes. Anteriormente la oferta era estandarizada, todas las empresas ofrecían servicios de mantenimiento y asistencia técnica, ya que la normativa estipulaba como condición obligatoria hacer uso de ellos para mantener la garantía de fábrica. Hoy en día, debido a las nuevas regulaciones normativas los clientes pueden justificar legalmente su no uso de los servicios de mantenimiento sin que esto signifique la pérdida de dicha garantía, lo que generó para estas empresas una baja considerable en la venta de servicios. Como consecuencia, son cada día más las empresas que destinan recursos al diseño de nuevos servicios que les permitan diferenciarse.

Axel Concesionarios de Imcruz es una empresa que vende vehículos nuevos en la ciudad de Sucre, al igual que la competencia, ha incrementado el volumen de ventas en los últimos años, apoyándose principalmente en la calidad de los productos que comercializa, sin embargo, actualmente los clientes hacen constantes reclamos por el servicio brindado, (Imcruz, 2018). Un análisis interno de la empresa refleja la importancia de elevar la atención en el asesoramiento y servicios posteriores. Se observa que el área de asistencia técnica y mantenimiento limita sus actividades a las instrucciones de su gerencia, sin embargo, no existe una coordinación permanente con el área de ventas, debilidad que se ve reflejada en reclamos constantes relacionados con estos servicios que oferta la empresa.

Se observa que la empresa centra su atención en sus productos y no así en servicios posteriores que permitan reforzar la fidelización de los clientes, se desconoce cómo fidelizar a los clientes del área de servicio posventa. En este contexto, el estudio científico que se presenta tuvo por objetivo diseñar una estrategia de fidelización para el área de servicios posventa para la empresa Axel Concesionarios de Imcruz en la ciudad de Sucre. 
Un modelo de servicio para el área de asistencia técnica y posventa beneficiará a Axel Concesionarios de Imcruz en la Ciudad de Sucre porque le permitirá conocer más a sus clientes, segmentarlos y hacer un análisis del mercado donde actúa, en lo que refiere a sus necesidades y expectativas, siendo la satisfacción y fidelización el objetivo principal a alcanzar como elemento central de calidad. De la misma forma el modelo de servicio puede constituirse en un elemento para reorganizar las tareas actuales de las áreas de venta y las demás involucradas, así como en su orientación hacia el cliente.

\section{Marco teórico}

La calidad es el conjunto de rasgos distintivos de un producto y/o servicio que influyen en la capacidad de satisfacer necesidades manifiestas o latentes, es la clave para la creación de valor y la satisfacción del cliente. La administración de la calidad es defender al cliente en la totalidad del proceso. El esfuerzo que una empresa destine para satisfacer a los clientes es bastante útil en la medida que la empresa se ajuste a los principios de la administración de la calidad total (Kotler, 2006). La calidad de productos y servicios, la satisfacción de los clientes y la rentabilidad de la empresa, están estrechamente relacionados (Zeithaml, 2009).

Administrar servicios es administrar momentos de verdad, es decir, cada momento en que el cliente toma contacto directo con el personal de la empresa y hace una evaluación consciente o inconsciente de la calidad del servicio. El total de las percepciones colectivas de todos los clientes crea la imagen de la calidad de servicio que se presta, (Lovelock, 2007). Un método para medir la satisfacción es el Modelo Servperf, que toma como base de su elaboración la teoría del Modelo Servqual. Su origen se debe a las críticas referidas a la alta probabilidad de sesgo en el modelo precedente, (Zeithaml, 2009). La aplicación del modelo plantea que la calidad del servicio es un antecedente de la satisfacción del cliente, a su vez, la satisfacción del cliente tiene un efecto significativo en su intención de compra y, por último, la calidad del servicio tiene menos efecto en la intención de compra que la satisfacción del cliente, (Ibarra, 2015; Cronin, 1994).

Se debe partir de los conceptos de calidad y satisfacción ya estudiados para poder determinar el alcance de lo que es fidelización. Los clientes satisfechos e incluso complacidos tienden a convertirse en apóstoles fieles de una empresa (Lovelock, 2007), el nivel de calidad del servicio ofertado con relación al entregado y la percepción que el cliente tenga determinará el nivel de éxito que tenga en que el cliente vuelva a requerir del servicio de la empresa. Se hace más difícil a los competidores atraer a un cliente leal que convertir a uno cuyo nivel de lealtad ha sido deteriorado por la falta de confianza en la empresa o por la hostilidad de sus empleados (Tschohl, 2010).

Por su parte, administración de relaciones con los clientes (ARC), es el proceso por medio del cual se establecen y mantienen las relaciones con los clientes, (Lovelock, 2007). Permite entender, segmentar y clasificar a los clientes, con el objetivo de dirigir mejor las promociones y hacer ventas complementarias, e incluso implementar acciones de alerta de deserción que indiquen si un cliente está 
en peligro de cambiar de empresa. La estrategia es un sistema integrado involucra cinco procesos fundamentales: a) desarrollo de estrategia, b) la creación de valor, c) integración multicanal, d) administración de la información y e) evaluación del desempeño, (Sales, 2013).

El marketing de bases de datos también se enfoca en las transacciones de mercado, pero incluye intercambio de información. El objetivo de esta herramienta es identificar y construir una base de datos de los clientes actuales y potenciales, enviar mensajes diferenciados a partir de las características y las preferencias de los consumidores, así como hacer un seguimiento de cada relación para supervisar el costo de adquirir al consumidor y el valor de las compras resultantes. La teoría referente al marketing relacional que se subdivide en el marketing de base de datos, marketing de interacción y marketing de redes; se consideró en este estudio en una herramienta base que permita estar en constante contacto con los clientes (Kotler, 2006).

\section{MÉTODOS}

$\mathrm{L}$ a investigación se desarrolló bajo un enfoque dialéctico, considerando que se estudió la realidad cambiante de una empresa en desarrollo. Tuvo alcance relacional ya que se encontró la relación entre la administración de la calidad de los servicios, satisfacción y fidelidad de los clientes.

La observación directa de forma sistemática y controlada permitió obtener información de carácter cualitativo relacionada al servicio prestado (se usó la guía de observación directa como herramienta). Las encuestas, con base en un cuestionario en escala Likert, se aplicaron para la recopilación de datos relacionados con las variables satisfacción y fidelización, permitieron registrar las percepciones y expectativas de los clientes sobre los servicios ofertados por la empresa. Por medio de las entrevistas (se usó la herramienta guía de entrevista), se logró conocer las proyecciones que tienen los propietarios y gerentes con relación al crecimiento de la empresa.

El Modelo Servperf se aplicó mediante un cuestionario, que incluye consultas por cada ítem (fiabilidad, seguridad, empatía, elementos tangibles y capacidad de respuesta), contiene 22 declaraciones que se midieron con escala de Likert de 5 , siendo 1 totalmente en desacuerdo y 5 totalmente de acuerdo. Asimismo, se diseñó otro cuestionario enfocado en medir la fidelización. Para la correspondiente prueba de hipótesis (para medir la relación entre fidelización y satisfacción) se utilizó la prueba Chi Cuadrado de Pearson.

Con base en la interpretación de resultados de la encuesta y de la entrevista, se determinó la conveniencia de una estrategia ARC como alternativa para incrementar la fidelización de los clientes actuales y atraer a nuevos hacia la empresa. La definición de la propuesta de estrategia de fidelización siguió la siguiente secuencia lógica: a) etapas de implementación, b) escenarios de acción según dimensiones Servperf y c) creación de valor (acciones a desarrollar). 


\section{RESULTADOS}

$\mathrm{U}$ na vez aplicados los instrumentos y sistematizados los resultados obtenidos, se advierte que las dimensiones capacidad de respuesta y fiabilidad son las que tienen mayor importancia asignada (35,15\% y $25,15 \%$ respectivamente), y a la vez generan menor nivel de satisfacción. Las dimensiones elementos tangibles y empatía demuestran irregularidad en los niveles de satisfacción, a diferencia de la dimensión seguridad.

Existe alta intención de solicitud de servicios posventa, la condición de garantía es el principal motivo de solicitud de servicios posventa. el $73 \%$ de clientes rechazan la prestación de servicios actuales, constituyéndose en la posible porción de clientes que dejarían de hacer uso de los servicios de la empresa. El rechazo de los servicios posventa de la empresa empieza desde el proceso de venta y se prolonga hasta los requerimientos posteriores.

Se consideró importante para el análisis general presentar el valor porcentual de cada ítem por cada dimensión del modelo Servperf (tabla 1).

Tabla 1. Relación nivel de satisfacción por dimensión e ítem.

\begin{tabular}{|c|c|c|c|c|}
\hline DIMENSION & ITEM & INSATISFACCION (\%) & SATISFACCION (\%) & TOTAL \% \\
\hline \multirow[t]{3}{*}{ Elementos tangibles } & Comodidad en instalaciones & 2,33 & 1 & 10,00 \\
\hline & Presentación y aseo del personal & 2,96 & 0,37 & \\
\hline & Facilidad de acceso y ubicación & 1,42 & 1,91 & \\
\hline \multirow[t]{3}{*}{ Empatía } & Comunicación entendible & 3,24 & 1,71 & 14,85 \\
\hline & Educación y respeto en trato al cliente & 2,56 & 2,39 & \\
\hline & Predisposición en atención al cliente & 3,45 & 1,50 & \\
\hline \multirow{3}{*}{$\begin{array}{l}\text { Capacidad de } \\
\text { respuesta }\end{array}$} & Flexibilidad en horarios de atención & 7,36 & 4,36 & 35,15 \\
\hline & Soluciones a problemas & 8,18 & 3,54 & \\
\hline & Soluciones a emergencias & 8,46 & 3,25 & \\
\hline \multirow[t]{3}{*}{ Fiabilidad } & $\begin{array}{l}\text { Entrega de servicio de acuerdo con } \\
\text { lo solicitado }\end{array}$ & 6,24 & 2,15 & 25,15 \\
\hline & Cuidado y previsiones en trato al vehículo & 6,26 & 2,12 & \\
\hline & Muestra de dominio de procedimientos & 4,71 & 3,67 & \\
\hline \multirow[t]{3}{*}{ Seguridad } & Honestidad personal posventa & 0,33 & 4,62 & 14,85 \\
\hline & Confianza en trato al cliente & 1,49 & 3,46 & \\
\hline & Cumplimiento de horarios y tiempos & 0,83 & 4,12 & \\
\hline
\end{tabular}

Según se muestra en la tabla 1, existe insatisfacción en todas las dimensiones excepto en la dimensión "seguridad". Este resultado demuestra que varias de las causales de insatisfacción son 
generadas por no atender y entender la necesidad del cliente. La situación actual de la empresa muestra un nivel de insatisfacción del $59.7 \%$ en los servicios posventa y un nivel de satisfacción del 40,3\%. La importancia relativa asignada por los clientes a cada dimensión es: elementos tangibles $10,00 \%$, empatía $14,85 \%$, capacidad de respuesta $35,15 \%$, fiabilidad $25,15 \%$ y seguridad $14,85 \%$.

El coeficiente de correlación aplicado, por las características de las variables nominales y ordinales, es la prueba Chi Cuadrado de Pearson (tabla 2). Para ello corresponde plantear la hipótesis nula (Ho) y la hipótesis alternativa $(\mathrm{Ha})$, de la siguiente forma:

Ho = La satisfacción de clientes en el área de posventa no tiene una relación directa con el nivel de fidelización hacía los servicios de la empresa.

$\mathbf{H a}=$ La satisfacción de clientes en el área de posventa tiene una relación directa con el nivel de fidelización hacía los servicios de la empresa.

Tabla 2. Análisis Chi Cuadrado para prueba de hipótesis.

\begin{tabular}{|c|c|c|}
\hline \multirow{2}{*}{$\begin{array}{l}\text { Variable Independiente: NIVEL DE } \\
\text { SATISFACCION DE LOS CLIENTES }\end{array}$} & \multicolumn{2}{|c|}{ Variable Dependiente: FIDELIZACION HACIA EL SERVICIO } \\
\hline & \multicolumn{2}{|c|}{ Dimensión: Recomendación de Servicios } \\
\hline \multirow{4}{*}{ Dimensión Elementos Tangibles } & Correlación Chi Cuadrado & 11,529 \\
\hline & Sig. (bilateral) & 0,01 \\
\hline & $\mathrm{N}^{\circ}$ de Elementos & 330 \\
\hline & Correlación Chi Cuadrado & 9,3 \\
\hline \multirow[t]{3}{*}{ Dimensión Empatía } & Sig. (bilateral) & 0,03 \\
\hline & $\mathrm{N}^{\circ}$ de Elementos & 330 \\
\hline & Correlación Chi Cuadrado & 21,456 \\
\hline \multirow[t]{3}{*}{ Dimensión Capacidad de Respuesta } & Sig. (bilateral) & 0,00 \\
\hline & $\mathrm{N}^{\circ}$ de Elementos & 330 \\
\hline & Correlación Chi Cuadrado & 44,688 \\
\hline \multirow[t]{3}{*}{ Dimensión Fiabilidad } & Sig. (bilateral) & 0,00 \\
\hline & $\mathrm{N}^{\circ}$ de Elementos & 330 \\
\hline & Correlación Chi Cuadrado & 10,776 \\
\hline \multirow[t]{2}{*}{ Dimensión Seguridad } & Sig. (bilateral) & 0,01 \\
\hline & $\mathrm{N}^{\circ}$ de Elementos & 330 \\
\hline
\end{tabular}

En la tabla 3 se planteó el valor de alfa ( $\alpha$ ) de 0,05 (nivel de error máximo aceptado) como nivel máximo de error. Se comprobó entonces que en las 5 dimensiones se debe rechazar la hipótesis nula planteada. 
Tabla 3. Prueba de hipótesis según dimensión.

\begin{tabular}{lcccc}
\hline \multicolumn{1}{c}{ DIMENSION } & $\begin{array}{c}\text { Valor de } \\
\text { Significancia (P) }\end{array}$ & $\begin{array}{c}\text { Nivel de } \\
\text { Relación }\end{array}$ & $\begin{array}{c}\boldsymbol{\alpha} \text { (Margen de } \\
\text { Error) }\end{array}$ & Interpretación \\
\hline Elementos Tangibles & 0,01 & $\leq$ & 0,05 & Se rechaza Ho \\
Empatía & 0,03 & $\leq$ & 0,05 & Se rechaza Ho \\
Capacidad de Respuesta & 0,00 & $\leq$ & 0,05 & Se rechaza Ho \\
Fiabilidad & 0,00 & $\leq$ & 0,05 & Se rechaza Ho \\
Seguridad & 0,01 & $\leq$ & 0,05 & Se rechaza Ho \\
\hline
\end{tabular}

En la tabla 4 se presenta una relación entre las principales causas de rechazo de los servicios actuales detectadas en el diagnóstico gracias a la aplicación de entrevistas y las dimensiones que capacidad de respuesta y fiabilidad (áreas en las que se deber priorizar acciones de mejora).

Tabla 4. Relación principales dimensiones y causas de insatisfacción.

\begin{tabular}{clll}
\hline DIMENSION & \multicolumn{1}{c}{ CAUSA 1 } & \multicolumn{1}{c}{ CAUSA 2 } & \multicolumn{1}{c}{ CAUSA 3 } \\
\cline { 2 - 5 } & $\begin{array}{l}\text { Explicación Superficial de } \\
\text { Garantía }\end{array}$ & $\begin{array}{l}\text { Ausencia de Servicios } \\
\text { Complementarios e } \\
\text { Incentivos }\end{array}$ & $\begin{array}{l}\text { Carencia de } \\
\text { Seguimiento Posventa }\end{array}$ \\
$\begin{array}{c}\text { Capacidad de } \\
\text { Respuesta }\end{array}$ & $\begin{array}{l}\text { La venta y atención al cliente } \\
\text { finaliza con entrega de } \\
\text { producto. }\end{array}$ & $\begin{array}{l}\text { Necesidad de programa de } \\
\text { fidelización con base en } \\
\text { registro de solicitudes y } \\
\text { visitas }\end{array}$ & $\begin{array}{l}\text { Personal de atención } \\
\text { exclusivo para taller y } \\
\text { horarios definidos. }\end{array}$ \\
\cline { 2 - 4 } Fiabilidad & $\begin{array}{l}\text { Ausencia de revisiones en } \\
\text { taller guiadas a clientes de } \\
\text { acuerdo con antecedentes. }\end{array}$ & $\begin{array}{l}\text { Información elemental } \\
\text { acerca del funcionamiento } \\
\text { del vehículo. }\end{array}$ & $\begin{array}{l}\text { Inexistencia de llamados } \\
\text { y seguimiento para } \\
\text { atención de consultas u } \\
\text { otros. }\end{array}$ \\
\hline
\end{tabular}

Así, en la tabla 4, para estudiar las causas de la insatisfacción se tomaron en cuenta las dos áreas en las que existe mayor insatisfacción y que además son las más relevantes para los clientes (capacidad de respuesta y fiabilidad). En relación con la dimensión "capacidad de respuesta" las principales oportunidades de mejora tienen relación con: horarios y personal de atención en el taller, creación de un programa de fidelización, oferta de servicios complementarios y atención postventa. Las principales oportunidades de mejora en relación con la dimensión "fiabilidad" son: atención y seguimiento a 
solicitudes, asesoramiento referido al funcionamiento del vehículo y atención personalizada en el taller (considerando historial del vehículo).

Este análisis complementario permite respaldar la estrategia propuesta a continuación.

\section{Propuesta de estrategia de marketing relacional de base de datos de control de calidad y fidelización}

La propuesta que se planteó "estrategia relacional de marketing de base de datos", se centró en prestar un servicio al cliente que lo fidelice y estimule a retornar a la empresa, para ello la empresa debe tomar la iniciativa en las acciones a realizar. La propuesta sigue la siguiente secuencia lógica: etapas de implementación, escenarios de acción según dimensiones Servperf y creación de valor (acciones a desarrollar).

De la misma forma, se considera los resultados obtenidos en el diagnóstico para poder respaldar la propuesta: a) alta intención de solicitud de servicios posventa, b) condición de garantía como principal motivo de solicitud de servicios posventa, correspondiente al $64 \%$, y $16 \%$ solicitado gracias al alto nivel de calidad del servicio percibido, c) dimensiones "capacidad de respuesta y fiabilidad", con mayor importancia asignada en un 60,3\% por los clientes, a la vez con menor nivel de satisfacción, 30,14\%, d) el $73 \%$ de clientes rechazan la prestación de servicios actuales, constituyéndose estos últimos en la cantidad de clientes que dejarían de hacer uso de los servicios de la empresa, e) el rechazo de los servicios posventa que ofrece la empresa inicia en el proceso de venta y se prolonga hasta los requerimientos posteriores.

\section{Etapas de implementación de propuesta}

Se plantean etapas que involucran al área de ventas como punto de contacto inicial con el cliente y como fuente de información para las demás áreas, en este caso para el área de posventa, seguimiento cronológico, servicios extraordinarios, y capacitaciones a consideración en la empresa entre otros.

Etapa 1: Proceso de compra- venta (cobertura de garantía)

Se propone en esta etapa que la empresa considere aplicarla en una explicación de lo que es el Servicio de Garantía, como responsabilidad de la empresa, y costos emergentes cargo del cliente. Como principal respaldo se sugiere elaborar una Carta de Bienvenida y Manual Guía Posventa propio del Concesionario.

\section{Etapa 2: Interacción área de venta a área posventa}

Acciones internas y generación de Base de Datos organizada que facilite el área de ventas, con datos del cliente, vehículo y condiciones de venta; para armado de File Digital Posventa y de respaldo de trabajo a realizar con el cliente a futuro. 


\section{Etapa 3: Seguimiento posventa}

Contacto (telefónico, mail u otro medio) con cliente, por personal posventa, referente al funcionamiento del vehículo, dudas y su satisfacción en lapsos de cinco a diez días, y programable cada treinta días. Así como la respuesta y asistencia a algunos requerimientos, cuyo costo no sea atribuible al cliente como ser explicaciones de funcionamiento de accesorios, cuidados preventivos, levantamiento de sugerencias y otros, que sean base para un futuro análisis.

\section{Etapa 4: Personal de asistencia técnica posventa}

Aplicado con el apoyo del personal posventa de taller a quien recurrir por algún medio de comunicación o transporte (sujeto a cobro), ante una eventual emergencia. Servicio programable vía turnos al personal técnico, considerando pueda o no haber sido requerido en su "turno". Proponiendo como material mínimo para otorgar al personal: a) set de Herramientas técnicas, b) teléfono celular corporativo, c) contactos de auxilio mecánico (remolque, técnicos y empresas aseguradoras), d) pago por servicio laboral extraordinario y/o compensación días de descanso y e) capacitaciones relacionadas a los puntos precedentes para toma de decisiones ante imprevistos.

El éxito de estas etapas dependerá de establecer registros ordenados cronológicamente con el objetivo de generar la de fidelización del cliente, base del futuro crecimiento de la empresa en posventa.

\section{Escenarios de Acción e Implementación de Estrategias}

En función al estudio realizado y según la teoría aplicada acorde al Modelo Servperf, la empresa va a encontrar escenarios, a ser planteados por los mismos clientes y que se los fue descubriendo a lo largo de la investigación, para los que se plantea una directriz de las acciones a realizar de acuerdo con la importancia de las dimensiones e ítems para los clientes y las posibilidades de la empresa.

Escenario 1: La "capacidad de respuesta", dimensión de mayor importancia para los clientes de la empresa y la menos atendida, por la que los clientes dejan de hacer uso de los servicios posventa, ya que no existe personal de consultas y atención de emergencias y/o. Se propone, asignar funcionarios de taller de atención durante horarios de trabajo y fuera de los mismos, con material y equipamiento que permitan asesorar al cliente que lo requiera ante una eventualidad. A ello se complementa la propuesta de capacitaciones periódicas que les permita conocer más de los vehículos, posibles fallas, la forma de solucionarlas y atención al cliente en esa situación. De la misma forma este personal debe tener como base información para asesorar al cliente en relación con los servicios de las aseguradoras, remolques, talleres depósitos o talleres de guarda de vehículos temporal, dependiendo del caso a atender.

Escenario 2: La "fiabilidad", como muestra de confianza del cliente en los servicios de la empresa, por lo que dentro la propuesta se considera aplicar el contacto directo con el cliente, en esta parte, en base a una explicación de los alcances de la garantía y la predisposición de asistencia posterior, de tal 
manera que sea el funcionario de asistencia posventa el intermediador en este escenario, esto se debe materializar con la información base a brindar por el área de ventas. Desde luego en este primer paso la Empatía, actualmente indiferente para los clientes, es base para generar un lazo de confianza a ser complementado por los demás funcionarios según las tareas que vayan siendo solicitadas por los mismos clientes.

Escenario 3: La dimensión "seguridad" surge como complemento a la anterior, ya que, si bien esta dimensión no es muy valorada por los clientes, la honestidad, confianza inspirada y cumplimiento de horarios garantizan la Capacidad de Respuesta y Fiabilidad ya señaladas.

Escenario 4: La dimensión "elementos tangibles" se constituyen en todos aquellos elementos perceptibles por los sentidos de cuyo resultado dependerá la imagen que quiere dar de la empresa y los servicios que esta ofrece. Esta percepción inicia desde que los clientes ingresan en instalaciones de la empresa y va aparejada con la dimensión empatía, comprendida por los siguientes aspectos: a) Señalética adecuada para que el cliente acceda a la oficina o ambiente correspondiente, orientará al interior de la empresa. b) Ambiente exclusivo de espera, con servicio de agua, café u otro dispensador para la espera, asientos e información (revistas, cuadros relacionados al tema vehicular y de la empresa). c) Espacio exclusivo en taller para la entrega del vehículo (mantenimiento, reparación o revisión) por personal correctamente uniformado y aseado, y el vehículo con algunas señales como forros o tapices que muestren al cliente el trato adecuado. d) El servicio solicitado en orden de trabajo original y en copia al cliente para realizar la respectiva verificación del trabajo solicitado. e) explicación del trabajo realizada de forma concreta y precisa.

Escenario 5: En el estudio se detectó como una causal relevante por la que los clientes dejan de hacer uso de los servicios la ausencia de incentivos, que reconozcan ese compromiso que tiene la empresa para con el cliente, que a la vez le hagan sentir participe y miembro fundamental de esta. En este sentido y después del análisis de los puntos anteriores se propone para la nueva área: generar relaciones basadas en recompensas con incentivos y recompensas con base en la frecuencia uso de los servicios, generando un valor para el cliente, orientado a hacerle sentir como parte de un grupo selecto. Al mismo tiempo conocer y reconocer a los clientes frecuentes de los servicios de la empresa, como parte de la administración del marketing de base de datos, anticipándose a sus requerimientos.

\section{Creación de valor para el cliente}

Con el fin de crear valor para el cliente, se propuso seguir 2 acciones: intensificar relaciones con los clientes y desarrollar acciones basadas en recompensas.

Intensificar Relaciones con los Clientes. El proceso de venta no concluye con la entrega del producto, más al contrario se debe entender como una obligación el conocer las expectativas y percepciones del cliente, para ello se plantea las siguientes acciones: a) Planificación de un contacto periódico con el cliente. b) Crear un "manual guía posventa del concesionario", que sea entregado al cliente en el momento de compra del vehículo. c) Prestación de servicio de aseo y limpieza para el 
producto, programable dentro los primeros 30 días de la entrega del vehículo y de acuerdo con las posibilidades de la empresa y el cliente. Haciendo participe al cliente de este servicio exclusivo.

Desarrollar relaciones basadas en recompensas. El análisis está orientado hacia un conjunto de elementos motivadores para los clientes, enfocados a incentivar su solicitud de servicios: a) Incentivo a solicitudes de servicios de mantenimiento, considerando la placa como factor de control (mantenimiento gratuito por una determinada cantidad de solicitudes de servicio). b) Descuentos demostrables en servicios, obsequios, bonos, vales e inclusive alianzas con otras empresas (ejemplo empresas aseguradoras, escuelas de conducción, empresas de lavado y aseo). c) Servicios de asistencia técnica y solicitudes de mantenimiento "puerta a puerta". d) Invitaciones y gestión de atenciones especiales a clientes frecuentes. e) Acciones que permitan conocer al cliente e incrementar la confianza a momento de solicitar los servicios. f) Incrementar el nivel de comunicación entre área de venta y posventa referente al file de un cliente y el proceso de venta. g) Presentación del técnico encargado, antes de que el vehículo ingrese a taller (considerar tratamiento similar al momento de la despedida). h) Personal posventa que permita atender requerimientos en horarios fuera de oficina. i) Habilitación de un servicio extra para demostración de vehículos, ante la frecuencia de observaciones y/o problemas recurrentes.

Todo lo expuesto en este apartado se resume en el gráfico 1, en él se puede ver la propuesta de estrategia de marketing relacional de base de datos de control de calidad y fidelización.

Acceso a información básica y datos de registro para armado de File Digital Posventa
Generar una base de datos que se inicia en proceso de compra-venta.
Programación y seguimiento Periódico para registrar en File Digital Posventa(Estadística)
Asistencia Técnica Básica

- Herramientas, celulares.

- Contactos, auxilio u otros 
ESTRATEGIAS SERVPERF POR ESCENARIOS AXEL CONCESIONARIOS SRL SUCRE

DIMENSION CAP. DE
RESPUESTA
- Personal exclusivo para
atención consultas y/o
reclamos.
- Personal de atención en
fuera de oficina los
mismos.
- Capacitaciones
periódicas.

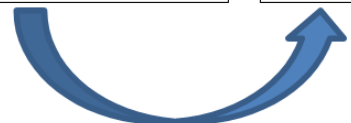

DIMENSION

FIABILIDAD Y

EMPATIA

- Explicación de alcance de garantía y personal de contacto.

- Guía de servicios de la empresa para solicitudes.

\section{DIMENSION \\ ELEMENTOS \\ TANGIBLES}

- Señalética en Ingreso.

- Espacios adecuados de espera.

- Formalismo, Manual guía de respaldo de servicio realizado.

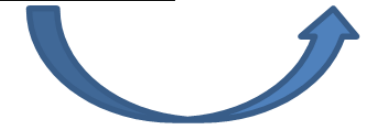

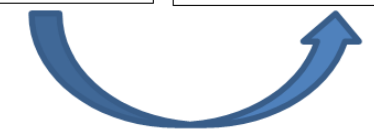

DIMENSION SEGURIDAD Y

EMPATIA

- Cumplimiento de tareas y respaldados por órdenes de trabajo con copia cliente.

\section{PLAN DE ACCIONES SEGÚN ESTRATEGIAS SERVPERF \\ $\sqrt{1}$

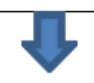

\section{INTENSIFICAR} RELACIONES CLIENTES

- Contacto Periódico Posventa.

- Creación Manual Guía AXEL.

- Prestaciones de servicios sorpresa (Ej. servicio de lavado)
RELACIONES BASADAS EN RECOMPENSAS POR FRECUENCIA DE USO

- Prestación de Servicios Gratuitos.

- Bonos, obsequios en accesorios y/o servicios.

- Invitaciones a ferias, reconocimientos, etc.

\section{RELACIONES PERSONALIZADAS}

- Creación de file Digital cliente.

- Presentación de técnicos a clientes.

- Técnico volante para asistencia técnica.

- Servicio demostración de fallas técnicas en vehículo.

Gráfico 1. Resumen estrategias y plan de acción marketing relacional.

En el gráfico 1 se observa un resumen de las estrategias que se plantearon (para cada dimensión considerada), partiendo de las bases del marketing relacional. En la parte inferior del gráfico se ve a grandes rasgos el plan de acción. 


\section{Proyecciones de mejora}

Se espera percibir mejoras en los niveles de satisfacción a partir del primer trimestre de aplicada la propuesta, considerando pertinente medir las mejoras trimestralmente aplicando el cuestionario Servperf a los clientes que tengan contacto con la empresa en ese periodo y comparar los resultados con los obtenidos en el momento cero, para poder verificar el alcance y la mejora de cada una de las dimensiones, así como las posibles acciones a replantear.

Tabla 5. Proyección de mejora según dimensión.

\begin{tabular}{|c|c|c|c|c|c|c|c|}
\hline \multirow[b]{2}{*}{ DIMENSION } & \multirow[b]{2}{*}{ Ítem } & \multirow[b]{2}{*}{ Valor } & \multirow{2}{*}{$\begin{array}{l}\text { Promedio } \\
\text { Por } \\
\text { Dimensión }\end{array}$} & \multicolumn{4}{|c|}{ Proyección de Satisfacción de clientes } \\
\hline & & & & $\begin{array}{c}\text { Trimestre } \\
1\end{array}$ & $\begin{array}{c}\text { Trimestre } \\
2\end{array}$ & $\begin{array}{c}\text { Trimestre } \\
3\end{array}$ & $\begin{array}{c}\text { Trimestre } \\
4\end{array}$ \\
\hline \multirow{4}{*}{$\begin{array}{l}\text { ELEMENTOS } \\
\text { TANGIBLES }\end{array}$} & 1 & 3,11 & & & & & \\
\hline & 2 & 2,47 & 3,03 & 3,48 & 4,00 & 4,60 & 5 \\
\hline & 3 & 3,50 & & & & & \\
\hline & 4 & 3,61 & & & & & \\
\hline \multirow[t]{2}{*}{ EMPATIA } & 5 & 4,31 & 3,48 & 4,00 & 4,60 & 5 & 5 \\
\hline & 6 & 2,51 & & & & & \\
\hline CAPACIDAD & 7 & 3,05 & & & & & \\
\hline DE & 8 & 3,00 & 3,00 & 3,45 & 3,97 & 4,56 & 5 \\
\hline \multirow[t]{2}{*}{ RESPUESTA } & 9 & 2,97 & & & & & \\
\hline & 10 & 3,08 & & & & & \\
\hline \multirow[t]{3}{*}{ FIABILIDAD } & 11 & 4,01 & 3,31 & 3,80 & 4,37 & 5 & 5 \\
\hline & 12 & 2,85 & & & & & \\
\hline & 13 & 4,54 & & & & & \\
\hline \multirow[t]{2}{*}{ SEGURIDAD } & 14 & 3,82 & 4,18 & 4,81 & 5,53 & 5 & 5 \\
\hline & 15 & 4,18 & & & & & \\
\hline
\end{tabular}

En la investigación se afirma que, en caso de implementar la estrategia, hacer las mediciones propuestas e implementar cambios si fuera pertinente, al cabo de 12 meses el cliente estaría completamente satisfecho. Se resalta la importancia de cumplir con el plan debido a que contempla las necesidades específicas de la empresa y cualquier variación que no sea estudiada con rigor científico podría impedir la consecución del objetivo planteado. 


\section{CONCLUSIONES}

$\mathrm{S}$ e concluye que la venta de vehículos nuevos puede potenciarse elevando el componente de servicios, diferenciándose así de la competencia y fidelizando a los clientes. En el contexto particular de la empresa Axel Concesionarios de Imcruz SRL Sucursal Sucre, se logró demostrar la relación directa entre satisfacción de servicios (en sus 5 dimensiones) y fidelización, a través de la aplicación del Modelo Servperf. Se estimó que es factible alcanzar un punto máximo de satisfacción de los clientes (en este caso el tiempo estimado es 12 meses), aplicando una estrategia de fidelización enmarcada en técnicas propias del marketing relacional de base de datos que, en este caso, sugieren seguir sistemáticamente 4 etapas: a) proceso de compra-venta (cobertura de garantía), b) interacción área de venta y área posventa, c) seguimiento posventa d) personal de asistencia técnica posventa.

Con el propósito de comprobar los resultados de la implementación de la estrategia de fidelización y para comprobar si las acciones propuestas están alcanzando las metas proyectadas, se desarrollará un análisis estadístico de acuerdo con las dimensiones Servperf. Este estudio servirá como instrumento de referencia para la empresa en el desarrollo de capacitaciones, reorganización de tareas y servicios adicionales entre otros, en el corto y mediano plazo. De ser necesario se podrá en práctica planes de contingencia con respuestas rápidas y soluciones a emergencias u observaciones notables.

La creación de valor para el cliente implica, entre otras acciones, contar con información completa de cada cliente, en el contexto estudiado, la recolección de información debe partir de la provisión de información del área de posventa con base en un programa informático específico, con datos generales como ser contrato y placa de control del vehículo, solicitudes por senvicios de mantenimiento, revisiones y/o observaciones, datos personales (demográficos, psicosociales y de comportamiento) del cliente, estadísticas de comportamiento con la empresa, referencias a otros clientes, percepción de calidad en cada momento de verdad.

\section{REFERENCIAS}

Cronin Jr, J., y Taylor, S. (1994). Servperf versus SERVQUAL: reconciling performance-based and perceptions-minus-expectations measurement of service quality. Journal of marketing

EAE Business School. (2017). España. EAE Business School; 2017 [Consultado Septiembre 16, 2017] ¿Qué es la fidelidad del cliente y porque es tan importante? Disponible

en http://www.eaeprogramas.es

Imcruz Comercial S.A. (2018). Manuales de Garantía. Bolivia

Kotler, P., y Keller, L. (2006) Dirección de Marketing. 12va Edición. México. Editorial Pearson
Lovelock, C., y Wirtz, J. (2007). Marketing de Servicios. 6ta Edición. México Editorial Pearson Education

Morales, E., y Medina, V. (2015). Aplicación del modelo Servperf en los centros de atención Telcel, Hermosillo: una medición de la calidad en el servicio. Contaduría y administración. México

Sales, M. (2013). Diagrama de pareto. EALDE Business School.

Serrano, A. (2013) La Importancia de la Fidelización del Cliente Actual. Puro Marketing [Revista de Internet] 2013; [Consultado Septiembre 12, 2017]. Disponible en http://www.puromarketing.com 
Tschohl, J. (2010) Atención al Cliente. Editorial Service Quality Institute. 8va. Edición. Estados Unidos.
Zeithaml, V.; Parasuraman, P., y Berry, L. (2009). Marketing de Servicios. España Editorial: Mac Graw Hill. 5ta Edición 